\title{
Three Common Mistakes in Modeling and Analysis of QoS of Service-Oriented Systems
}

\author{
Vladimir Tosic ${ }^{1,2,3}$ \\ ${ }^{1}$ NICTA $^{*}$ \\ ${ }^{2}$ University of New South Wales, Sydney, New South Wales, Australia \\ ${ }^{3}$ University of Western Ontario, London, Ontario, Canada \\ vladat@computer.org
}

\begin{abstract}
Since the basic Web service technologies did not address QoS issues, a huge body of academic and industrial works studied various aspects of QoS modeling/specification, analysis, monitoring, and control for service-oriented systems. Unfortunately, some of these works adopted oversimplifications that are often not appropriate for the complex reality. In this discussion session, we will point out to three such recurring oversimplifications: 1) specifying provider's QoS guarantees without limiting the number of requests; 2) using past QoS measurements to predict future QoS without taking into consideration context of requests; and 3) predicting response time of a sequence of services as a simple addition of their response times without discussing circumstances under which such calculation is valid/invalid. A significant number of authors (often independently from each other) used these oversimplifications without informing readers about their limitations and consequences. We will discuss why such oversimplifications are mistakes and "anti-patterns" in QoS modeling and analysis for service-oriented systems.
\end{abstract}

Keywords: Quality of service, contract, performance analysis, performance prediction, Web service selection, Web service composition, anti-pattern.

* NICTA is funded by the Australian Government as represented by the Department of Broadband, Communications and the Digital Economy and the Australian Research Council through the ICT Centres of Excellence program. 